\title{
A Review: extraction of solar cell modelling parameters
}

\author{
Rituraj Tamrakar ${ }^{1}$, Archana Gupta ${ }^{2}$ \\ M.Tech Research Scholar, Dept., of Electronics and Telecommunication, Bhilai Institute of Technology, Durg, India ${ }^{1}$ \\ Professor, Department of Electrical Engineering, Bhilai Institute of Technology, Durg, India ${ }^{2}$

\begin{abstract}
Energy from the sun is the best option for electricity generation as it is abundantly available everywhere and sustainable source. Advance of Photovoltaic (PV) technology in recent years has made solar energy one of the practical alternative energy sources available in the energy market. In order to make the PV energy more affordable and cost effective, major focus of the research community and industry is improvement on power efficiency of PV systems. Parameter extraction of solar cell plays a crucial role in the simulation and design calculation of PV system. The circuit model of a solar cell is non-linear and transcendental. Different methods under the categories of analytic, iterative, and evolutionary computational have been proposed to extract the parameters of solar cell in recent years. This paper presents a survey on parameter extraction of solar cell model and its recent advances.
\end{abstract}

Keywords: Solar cell models, parameter extraction, analytical methods, iterative methods, evolutionary methods, optimization.

\section{INTRODUCTION}

Renewable energy sources are getting added attention in recent years as alternative means of generating electricity in various parts of the world. Various motivations are promoting serious contribution of environmental friendly energy sources in mass electricity production in many countries. Some of these reasons are: environmental concerns due to greenhouse effect, possible depletion and price increase of conventional energy primary resource. Solar energy is one of the most promising emission free resources that is currently being used worldwide to contribute to meeting rising demands of electric power. Solar photovoltaic system is not only providing reliable and environmentally friendly energy but also could economically visible for rural and remote areas as an only source of energy. Despite the challenges of variability of electricity generation, life duration of solar photovoltaic devices and economic feasibility of deployment, it is gaining significant attention. This is due to the fact that solar photovoltaic is a semiconductor device that produces DC electricity when sunlight shines on the photovoltaic. It is static, quite, and free of moving parts system without any gas or toxic emissions and therefore requires little operation and maintenance costs [1].

The solar cell is the elementary building block of the photovoltaic device. Solar photovoltaic cells are normally arranged into arrays called panel or a module which directly converts sun radiation into electricity. Solar cells are made of semiconductor material with a $\mathrm{p}-\mathrm{n}$ junction that has the property of, when exposed to sunlight, produces direct-current electricity proportional to the solar radiation $[2,3]$.

A non-linear current-voltage (I-V) characteristic exists in solar cell's behavior. Various representations have been proposed to describe the current-voltage relationship (I-V) in solar cells [4-7]. Lumped parameter equivalent circuit model is commonly used to simulate its behavior under different operating conditions. In practice, there are two main equivalent circuit models used to describe the nonlinear I-V relationship: single and double diode models. However, no matter what kinds of models, there are important PV parameters that need to be accurately extracted for the simulation, design, performance evaluation, and control of solar cell systems. The main parameters that describe solar cell models behavior are the generated photocurrent, saturation current, series resistance, shunt resistance, and diode ideality factor. The electrical current produced by a solar cell depends on the intensity of the incident light and on its intrinsic properties. An accurate estimation of the cell parameters required for accurate performance evaluation [8].

\section{SOLAR CELL MODELING}

Providing a mathematical model that accurately describes the electrical behavior of solar cell is quite crucial. Many equivalent circuit models have been developed and proposed to describe the I-V characteristics of solar cell. In practice, two commonly used models are single and double diode models [9].

\section{A. $\quad$ Single Diode Model}

Due to simplicity and accuracy, the single diode model is considered. The single diode five parameter model, which include a current source, one diode and two resistors is shown in fig. 1

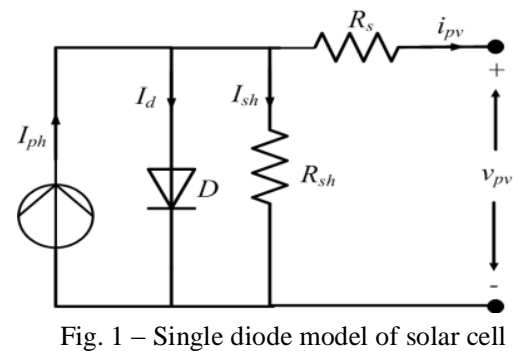


The output current of the cell is calculated as follows:

$i_{p v}=I_{p h}-I_{0}\left[\exp \left(\frac{v_{p v}+i_{p v} R_{s}}{a V_{t}}\right)-1\right]-\left(\frac{v_{p v}+i_{p v} R_{s}}{R_{s h}}\right)$

Where $I_{p h}$ is cell generated photocurrent, $I_{0}$ is reverse saturation current of diode, $V_{t}(k \mathrm{~T} / q)$ is the thermal voltage of the solar cell, $k$ is the Boltzmann constant $\left(1.380650 \times 10^{-23} \mathrm{~J} / \mathrm{K}\right), \quad q$ is the electron charge $\left(1.602176 \times 10^{-19} \mathrm{C}\right)$, and $\mathrm{T}$ is the temperature of the $\mathrm{p}-\mathrm{n}$ junction in Kelvin. Variables $a$ is the diode ideality constants, $R_{s}$ and $R_{s h}$ are series resistance and shunt resistance respectively and $v_{p v}$ is output voltage of the cell. For the single diode model, five parameter to be extracted are parameters $I_{p h}, I_{0}, R_{s}, R_{s h}$, and $a$. An accurate knowledge of the parameters of solar cells is necessary for the design, control of solar cell and process optimization [10]

\section{B. $\quad$ Double Diode Model}

Another way to describe solar cell electrical behavior is to model it as current source connected in parallel with a rectifying diode. In practice the current source is also shunted by another diode that models the space charge recombination current and a shunt leakage resistor to account for the partial short circuit current path near the cell's edges due to the semiconductor impurities and nonidealities. Moreover, solar cell metal contacts and semiconductor material bulk resistance are represented by a resistor connected in series with the cell shunt elements [11]. The equivalent circuit for this model is shown in Fig. 2.

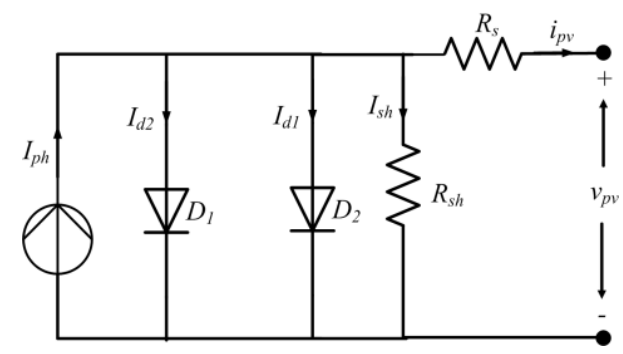

Fig. 2 - double diode model of solar cell

The output current of the solar cell can be formulated as:

$i_{p v}=I_{p h}-I_{d 1}-I_{d 2}-I_{s h}$

Where $I_{p h}$ is the cell generated photocurrent, $I_{d l}$ and $I_{d 2}$ are the first and second diode currents, respectively. $I_{s h}$ is shunt resistor current. The two diode currents $I_{d l}$ and $I_{d 2}$ and shunt current $I_{s h}$ can be calculated as,

$$
\begin{aligned}
& I_{d 1}=I_{01}\left[\exp \left(\frac{v_{p v}+i_{p v} R_{s}}{a_{1} V_{t}}\right)-1\right] \\
& I_{d 2}=I_{02}\left[\exp \left(\frac{v_{p v}+i_{p v} R_{s}}{a_{2} V_{t}}\right)-1\right] \\
& I_{s h}=\left(\frac{v_{p v}+i_{p v} R_{s}}{R_{s h}}\right)
\end{aligned}
$$

$I_{01}$ and $I_{02}$ are reverse saturation currents of diode1 and diode2 respectively, $a_{1}$ and $a_{2}$ are the diode ideality constants. The complete physical behavior of double diode model of solar cell is in relation with seven parameters $I_{p h}$, $I_{01}, I_{02}, R_{s}, R_{s h}, a_{1}$ and $a_{2}$.

\section{PROBLEM FORMULATION}

It is noted that current equation is nonlinear transcendental function. In order to extract the parameters of different solar cell models from the I-V data using the optimization techniques, we first need to define the objective function to be optimized [12]. In this work the root mean square error is used as the objective function, which is described as

$\varepsilon=\sqrt{\frac{1}{N} \sum_{k=1}^{N} f_{k}\left(v_{p v}, i_{p v}, \emptyset\right)^{2}}$

Where $\mathrm{N}$ is the no. of experimental data, $\emptyset$ is decision vector which consists of the parameters to be extracted [3].

In case of single diode model the function $f\left(v_{p v}, i_{p v}, \emptyset\right)$ is given by

$$
\begin{aligned}
& f\left(v_{p v}, i_{p v}, \emptyset\right)=I_{p h}-I_{0}\left[\exp \left(\frac{v_{p v}+i_{p v} R_{s}}{a V_{t}}\right)-1\right] \\
& -\frac{v_{p v}+i_{p v} R_{s}}{R_{s h}}-i_{p v} \\
& \emptyset=\left\{I_{p h}, I_{0}, R_{s}, R_{s h}, a\right\}
\end{aligned}
$$

For the double diode model, the function $f\left(v_{p v}, i_{p v}, \emptyset\right)$ is given by

$$
\begin{aligned}
& f\left(v_{p v}, i_{p v}, \emptyset\right)=I_{p h}-I_{01}\left[\exp \left(\frac{v_{p v}+i_{p v} R_{s}}{a_{1} V_{t}}\right)-1\right] \\
& \quad-I_{02}\left[\exp \left(\frac{v_{p v}+i_{p v} R_{s}}{a_{2} V_{t}}\right)-1\right]-\frac{v_{p v}+i_{p v} R_{s}}{R_{s h}}-i_{p v} \\
& \emptyset=\left\{I_{p h}, I_{01}, I_{02}, R_{s}, R_{s h}, a_{1}, a_{2}\right\}
\end{aligned}
$$

IV.

\section{LITERATURE REVIEW}

The accuracy of PV module mainly depends on the accuracy of the PV module models and the extraction methods being used to determine the model's parameters. Several parameter extraction methods have been proposed in recent years and some of contributions are being reviewed here. These methods can be classified into three categories such as analytic, iterative and evolutionary computational methods [13].

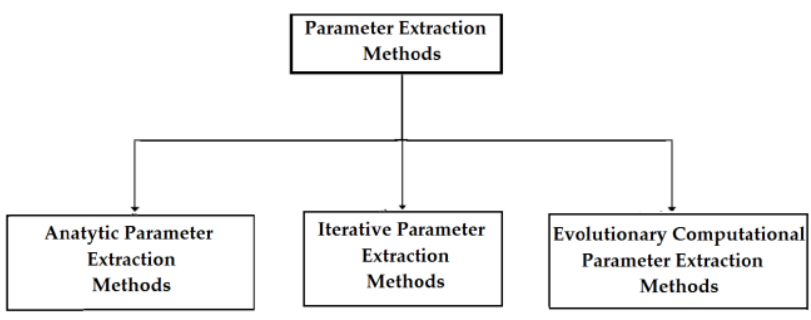

Fig 3 - Classification of parameter extraction methods of the PV panel 
A. Analytical Parameter Extraction Methods :

Analytical methods are common approaches in estimating the parameters by mathematical equations. One of the important features of the analytical method is its rapid calculation and reasonably accurate results. Analytical methods of parameter extraction are very simple and need very less computational time as only a single iteration is required for this.

M. de Blas et al. proposed a simple analytical method. In this method the cell model is constructed without considering the magnitude of $\mathrm{R}_{\mathrm{sh}}$. Hence, it is applicable to four-parameter model only [14].

Several methods were proposed with the use of Lambert W-function in parameter extraction of solar cell modeling. A. Jain and A. Kapoor presented Lambert Wfunction to express the transcendental current-voltage characteristic containing parasitic power consuming parameters like series and shunt resistances [15]. However, Current equation cannot be solved explicitly using elementary functions. J. Cubas et al. presented a method based on analytical formulation which turned the series resistor equation explicit by use of the Lambert Wfunction. The method was used to analyze commercial solar panel performance (i.e., the current-voltage- $I-V$ curve) at different levels of irradiation and temperature [16]. A. O. Conde et al. proposed method based on the Lambert W-function. In this the Co-content function was calculated from the exact explicit analytical expressions first and then the model parameters were extracted [17]. However, the explicit analytic expressions derived from Eq. (1) still remain unsuitable for extracting the model parameters. C. Zhang et al. proposed a simple fitting method to estimate all the parameters of a solar cell based on the Lambert $\mathrm{W}$ function. This method was used to analyze various solar devices, including $\mathrm{Si}$ solar cells, $\mathrm{Si}$ solar modules, standalone organic solar cells, tandem organic solar cells, multi-junction organic solar cells, and dye-sensitized solar cells (DSSCs) [18].

A. Laudani et al. presented a method which exploited the reduced forms of the five-parameter model. The five parameter of the model were expressed in independent and dependent unknowns through mathematical consideration, in order to reduce the dimensions of the search space. Hence, provides strong benefits in terms of convergence, computational costs and execution times [19].

M. R. AlRashidi et al. proposed a Pattern search (PS) optimization technique for estimating the solar cell. PS was a derivative free method which gave the flexibility to deal with objective functions that were not necessarily continuous, convex or differentiable. Advantages of this optimization algorithm were concept simplicity, ease of implementation, and computational efficiency [9].

A. Jain et al. compared previous work [15] with another analytical method using special transfunction theory (STFT) to calculate the parameters of a solar cell. STFT method enables the use of the whole I-V characteristics and produced results without any approximations. STFT had a lesser calculation time and gave a more accurate result in comparison to the Lambert W-function [20].

H. Al-Hamidi Focused on the estimation of the parameters of single-diode photovoltaic cell using piecewise approximation. A lumped circuit current-voltage characteristics model was used to represent photovoltaic cell. The model was approximated by a piecewise linear function that related the cell current and voltage with some parameters to be estimated [3].

Q. X. Jia et al. introduced analytical approach to calculate the solar cell parameters based on the lumped single exponential model. In this approach the diode junction ideality factor has been considered as an output characteristic dependent parameter under illuminated conditions [21].

J. Ma and K. L. Man proposed a fast least-squares approximation method for determining electrical characteristics of a PV Rp-model. This method was able to assess the electrical characteristics of an Rp-model under any operating temperature and solar irradiance. Test results had demonstrated that the proposed method reduced the computational effort of a single-diode PV model by around $40 \%$ while its accuracy is similar to precise analytical models [22].

Analytical methods perform efficiently at standard testing conditions (STC) for some models but these methods are found to be unsuitable for solar model for wide range of changing weather conditions. Because, the mathematical model of PV panel represented is implicit in nature [10].

\section{B. Iterative Parameter Extraction Methods:}

A number of iterative methods are available in the past few years.

R. Khezzar et al. compared three different methods, the slope method based on a geometric calculation, the explicit method based on an analytical solution and the iterative method based on a numerical resolution to estimate the parameters of the solar cell. However, $\mathrm{R}_{\mathrm{sh}}$ was neglected and rest four parameters were considered as its high value has a little contribution in the model [23].

A. Mohapatra et al. proposed trust-region-doleg method and compared with Villalva's iterative method. The trustregion-doleg method extracted all five parameters where as Villalva's iterative method extracted only two parameters $R_{s}$ and $R_{p}$ [24].

Newton-Raphson method (NRM) is one of the best rootfinding methods. Hence, most of the previous parameter extraction works such as [25-29, 30] were based on NRM. However, the improper choice of the initial conditions affects its accuracy and convergence.

F. Ghani et al. provided a method that calculated the values of series and shunt resistances numerically using the Newton-Raphson algorithm based on the Lambert Wfunction. Hence, the current could be expressed explicitly in terms of voltage and also for its relatively straightforward differentiation [31].

However, in these NRM algorithm based methods, five independent equations are necessary for extraction of the five unknown parameters. Also the Computation of a Jacobian matrix is required in the NRM algorithm. This Jacobian matrix consists of twenty-five numbers of single and double-derivative terms, which makes NRM very complex, lengthy and error prone [10, 13]. Another problem with all NRM methods is singularity problem which is division by zero may arise if initial conditions of 
the parameters are chosen improperly. Also, these NRM methods do not consider the boundary limits of the parameters $R_{s}, R_{s h}$ and $a$. A lot of assumptions are required in these methods in order to simplify the five parameter extraction problem which results in low value of $R_{S}$ and high value of $R_{s h}$ [2] denoting the ideal conditions of PV module. Hence, the parameters so obtained by these NRMs are found to be incorrect $[10,13]$.

The singularity problem is resolved in a parameter extraction method named as comprehensive parameter extraction method [13, 32]. This method is suitable to solve parameter extraction problem accurately at STC but may fail in other weather conditions, because it assumed values of $\mathrm{R}_{\mathrm{s}}, \mathrm{R}_{\mathrm{sh}}$ and $a$ independent of weather conditions and partial shedding conditions $[10,13]$.

\section{C. $\quad$ Evolutionary Parameter Extraction Methods:}

To resolve issues with analytical and iterative methods, Evolutionary computational algorithms which use artificial intelligence technique has received considerable attention for solar cell parameter extraction recently. These evolutionary algorithms are global optimization techniques. Evolutionary algorithms use iterative progress, such as growth or development in a population. This population is then selected in a guided random search using parallel processing to achieve the desired end. Such processes are often inspired by biological mechanisms of evolution. Some of the popular Evolutionary algorithm like Genetic algorithms (GAs) [6, 33], Particle Swarm Optimization (PSO) [7, 34, 35], Simulated annealing (SA) $[11,36]$, Artificial bee colony (ABC) [37, 38], Mimetic algorithm, Cuckoo search [39, 40], Bacterial Forging Optimization (BFO) $[10,41]$ etc. were applied to extract the parameter of solar cell.

Genetic algorithms perform a search by evolving a population of candidate solutions through the use of a nondeterministic operator. GA was originally developed for binary search spaces, but optimization over real-valued search-spaces is also possible. M. Zagrouba et al. proposed a numerical technique based on genetic algorithms to identify the electrical parameters of photovoltaic (PV) solar cells and modules [33]. However, Genetic algorithms become very inefficient if the fitness function is not decomposable, i.e. its parameters are not independent. Also, Genetic algorithms may have a tendency to converge towards local optima or even arbitrary points rather than the global optimum.

J. K. Maherchandani et al. proposed the hybrid Genetic algorithm, which was based on Genetic Algorithm and Nelder-Mead (NM) simplex search method in which GA generates the solutions for the new population and then the Nelder-Mead technique is used to improve the best solution which exists in the new population. Hybrid GANM algorithm combined the advantages of each algorithm in away to avoid their disadvantages [42].

Some of the population based algorithms are based on swarm intelligence. Among these, Particle swarm optimization (PSO) is best suited for continuous optimization which is inspired by social behavior of bird flocking or fish schooling. Population members are referred to as particles, which fly through the search space and evaluate the fitness values of visited points. The direction and velocity (step size) of each particle is affected by two attractors: the best location the particle has personally visited this far, and the best known point located by any particle in a certain neighborhood [43].

H. Qin and J. W. Kimball presented a swarm intelligence approach to extract equivalent circuit parameters of Photovoltaic Cells. The proposed method was applied to extract PV parameters from field test data, which came from a PV sensor array. One thousand sampled data were arbitrarily selected and used as training input for PSO [35]. M. Ye et al. applied particle swarm optimization to extract the solar cell parameters from illuminated currentvoltage characteristics and compared performance of the PSO with the genetic algorithms for the single and double diode models [7].

C. Saravanan and M. A. Panneerselvam proposed an improved model approach to single diode PV model by Hybrid Genetic Algorithm Particle Swarm Optimization (Hybrid GA-PSO) technique. Hybrid approach of GA and PSO is purposely chosen to utilize their effectiveness in these kinds of applications [34]. W. Huang et al. presented a chaotic particle swarm optimization algorithm (CPSO) for extracting solar cell model parameters, in which the global search performance and local convergence of particle swarm optimization (PSO) were improved by introducing a chaos search. The CPSO searched for optimal parameters without strict limitation on the search ranges [44].

Simulated Annealing (SA) is a random-search technique which exploits an analogy between the way in which a metal cools and freezes into a minimum energy crystalline structure (the annealing process) and the search for a minimum in a more general system. M. R. AlRashidi et al. presented Simulated Annealing based approach for optimal extraction of Photovoltaic characteristics [1, 11, 36]. SA's major advantage over other methods is an ability to avoid becoming trapped in local minima. Simulated annealing can deal with highly nonlinear models, chaotic and noisy data and many constraints.

M. Ketkar and A. M. Chopde presented Artificial Bee Colony (ABC) Algorithm for the extraction of the parameters. The algorithm is composed of three important elements: employed and unemployed foragers, and food sources. ABC has several features such as simplicity of implementation, promising optimization capability, fewer control parameters, etc. In this paper author have implemented various methods suggested to boost traditional $\mathrm{ABC}$ performance to solve multi parameter optimization problem [37].

Cuckoo Search (CS) is a nature-inspired optimization algorithm based on the inspiration of brood parasitic behavior of some cuckoo species in combination with the Levy flight behavior. J. Ma et al. proposed a CS-based parameter estimation method to extract the parameters of solar models [39]. R. Jovanovic et al. combined CS with the Nelder-Mead method. This makes it possible to use the flip operation introduces in the Nelder-Mead algorithm instead of the Levy flight which is a standard part of the CS. In this way, the hybridized algorithm becomes more 
robust and less sensitive to parameter tuning which exists in CS [40].

Bacterial Forging Optimization (BFO) is a heuristic search technique which is based on modeling of bacteria E. coli behavior present inhuman intestine. There are mainly four stages in a BFO optimized process such as chemotactic, swarming, reproduction and elimination and dispersal [41]. B. Subudhi and R. Pradhan presented an approach based on BFO for improving the extracting accuracy and computational time burden for extraction of PV module parameters and compared with NRM and PSO [10].

W. Han et al. proposed parameter extraction method based on artificial fish swarm algorithm (AFSA), which is originally inspired by the simulation of collective behavior of real fish swarms. AFSA has many advantages, including good global convergence, strong robustness, insensitivity to initial values, and simplicity in implementing. In addition to the regular operation, a mutation operator is designed to enhance the searching performance of the algorithm [45].

$\mathrm{X}$. Yuan et al. proposed another optional method, Chaotic asexual reproduction optimization (CARO) using chaotic sequence for global search is applied to this parameter extraction problem [46]. A. Askarzadeh and A. Rezazadeh proposed harmony search (HS) based parameter identification methods to identify the unknown parameters of the solar cell models. Simple concept, easy implementation and high performance are the features of HS method [47].

Fuzzy logic control has emerged as a powerful tool in recent years. A Knowledge-based fuzzy control is the rulebased fuzzy control, where the human knowledge is approached by means of linguistic fuzzy rules in the form if-then, which describes the control action in a special condition of the system. T. F. Elshatter et al. introduced a new type of data analysis that expresses these parameters by means of a fuzzy regression model. A fuzzy model has been developed for the PV arrays [48]. T. Bendib et al. presented a new solar cell parameters extraction tool, based on fuzzy control techniques [49].

The neural network is well known for its learning ability and approximation to any arbitrary continuous function. E. Karatepe et al. presented a neural network based approach for improving the accuracy of the electrical equivalent circuit of a photovoltaic module [50]. A. Kulaksz combined the advantages of neural networks, such as having a black-box model, and the linguistic interpretability of a fuzzy inference system and applied adaptive neuro-fuzzy inference system (ANFIS) to obtain three of the parameters in a single diode model of PV cells, namely series resistance, shunt resistance, and diode ideality factor. In this method the equivalent parameters can be obtained for a wide range of PV modules of different types using easily obtainable electrical parameters [51].

\section{CONCLUSION}

Different methods proposed in recent years for parameter extraction of a solar cell were reviewed. These methods were used to estimate different model parameter such as cell generated photocurrent, reverse saturation current, series resistance, shunt resistance, and the diode ideality constant that govern the current-voltage relationship of a solar cell. The existing parameter extraction methods were divided into three groups such as analytic, iterative and evolutionary computational methods. The analytic methods required less computational time for parameter extraction due to their simple structure. However, these were unable to find all the parameters of solar model. Iterative methods based on NRM were probably good option because of its accuracy and fast convergence, However NRM suffered from singularity problem. Also the convergence and accuracy were dependent on number of iterations. Again analytic and iterative methods were not suitable for fast varying weather conditions and partial shedding conditions. Evolutionary algorithms were the population based global optimizations algorithm. Many proposed Evolutionary methods concluded that these methods

were best suited for fast varying weather conditions and partial shedding conditions, however, these methods may suffer from the problem of premature convergence due to improper selection of control parameters and initial conditions.

\section{REFRENCES}

[1] K. M. El-Naggar, M. R. AlRashidi, M. F. AlHajri and A. K. AlOthman, "Simulated Annealing algorithm for photovoltaic parameters identification," Solar Energy, vol. 86, no.1, pp. 266274, 2012.

[2] M. G. Villella and J. R. Gazoli, "Comprehensive approach to modeling and simulation of photovoltaic arrays," IEEE Transactions on Power Electronics, vol. 24, no. 5, pp. 1198-1208, 2009.

[3] H. Al-Hamadi, "Estimation of Photovoltaic Cell Parameters Using Piecewise Linear Approximation," International Journal of Computer Science and Electronics Engineering, vol. 2, no. 1, pp. 2320-4028, 2014.

[4] M. K. Munji, W. Okullo, E. E. Van Dyk and F. J. Vorster, "Local device parameter extraction of a concentrator photovoltaic cell under solar spot illumination," Solar Energy Materials and Solar Cells, vol. 94, no. 12, pp. 2129-2136, Dec. 2010.

[5] W. Xiao, M. G. J. Lind, W. G. Dunford and A. Capel, "Real-time identification of optimal operating points in photovoltaic power systems," IEEE Transactions on Industrial Electronics, vol. 53, no. 4, pp. 1017-1026, 2006.

[6] N. Moldovan, R. Picos and E. G. Moreno, "Parameter extraction of a solar cell compact model using genetic algorithms," IEEE proceedings of the 2009 Spanish Conference of Electron Devices, Feb 2009, pp. 379-382.

[7] M. Ye, X. Wang and Y. Xu, "Parameter extraction of solar cells using particle swarm optimization," Journal of Applied Physics, vol. 105, no. 9, May 2009.

[8] M. Wolf, G. Noel and R. Stirn, "Investigation of the double exponential in the current-voltage characteristics of silicon solar cells," IEEE Transactions on Electron Devices, vol. 24, no. 4, pp. 419 - 428, April 1977.

[9] M. R. AlRashidi, K. M. El-Naggar and M. F. AlHajri, "Heuristic Approach for Estimating the Solar Cell Parameters," Recent Researches in Applied Information Science, pp. 80-83, Published by WSEAS Press, 2012.

[10] B. Subudhi and R. Pradhan, "Development of new parameter extraction scheme and maximum power point tracking system," PHD thesis, National Institute of Technology, Rourkela, July 2014.

[11] M. R. AlRashidi, K. M. El-Naggar and M. F. AlHajri, "Solar Cell Parameters Estimation Using Simulated Annealing Algorithm," world academy of science, engineering and technology, vol. 7, no. 4, pp. 149-152, 2013. 
[12] M. F. AlHajri, K. M. El-Naggar, M. R. AlRashidi and A. K. AlOthman, "Optimal extraction of solar cell parameters using pattern search,” Renewable Energy, vol. 44, pp. 238-245, 2012.

[13] B. Subudhi and R. Pradhan, "Characteristics Evaluation and Parameter Extraction of a Solar Array Based on Experimental Analysis," IEEE international conference on Power Electronics and Drive System (PEDS), December 2011, pp. 340-344.

[14] M. de Blas, J. Torres, E. Prieto and A. Gracia, "Selecting a suitable model for characterizing photovoltaic devices," Renewable Energy, vol. 25, no. 3, pp. 371-380, 2002.

[15] A. Jain and A. Kapoor, "Exact analytical solutions of the parameters of real solar cells using Lambert W-function," Solar Energy Materials and Solar Cells, vol. 81, no. 2, pp. 269-277, Feb.2004.

[16] J. Cubas, S. Pindado and C. de Manuel, "Explicit Expressions for Solar Panel Equivalent Circuit Parameters Based on Analytical Formulation and the Lambert W-Function," Energies, vol.7, pp. 4098-4115, 2014

[17] A. Ortiz-Conde, F. J. García Sanchez and J. Muci, "New method to extract the model parameters of solar cells from the explicit analytic solutions of their illuminated I-Vcharacteristics," Solar Energy Materials and Solar Cells, vol. 90, no.3, pp. 352-361, 2006.

[18] C. Zhang, J. Zhang, Y. Hao, Z. Lin and C. Zhu, 'A simple and efficient solar cell parameter extraction method from a single current-voltage curve," Journal of applied physics, vol. 110, no. 6, 2011

[19] A. Laudani, F. Riganti Fulginei and A. Salvini, "High performing extraction procedure for the one-diode model of a photovoltaic panel from experimental I-V curves by using reduced forms," Solar Energy, vol. 103, pp. 316-326, 2014.

[20] A. Jain, N. S. Singh and A. Kapoor, "An Exact Analytical Method for Calculating the Parameters of a Real Solar Cell Using Special Trans Function Theory(STFT)," International Journal of Renewable Energy, vol.3, no.1, pp. 201-206, 2012.

[21] Q. X. Jia, K. Ebihara and T. Ikegami, "Analytical solution for solar cell model parameters from illuminated current-voltage characteristics," Philosophical Magazine B, vol. 72, no. 4, pp. 375382, 1995.

[22] J. Ma, K. L. Man, T. O. Ting, N. Zhang, E. G. Lim, S. Guan, P. W. H. Wong, T. Krilavicius, D. Saulevicius and C. U. Lei, "Simple Computational Method of Predicting Electrical Characteristics in Solar Cells," Electronics and Electrical Engineering, vol. 20, no. 1, pp. 41-44, 2014.

[23] R Khezzar, M. Zereg, and A. Khezzar, "Comparative Study of Mathematical Methods for Parameters Calculation of CurrentVoltage Characteristic of Photovoltaic Module," IEEE international conference on Electrical and Electronics Engineering (ELECO), Nov. 2009, pp. 24-28

[24] A. Mohapatra, B. K. Nayak and K. B. Mohanty, "Comparative study on single diode photovoltaic module parameter extraction methods," IEEE International Conference on Power, Energy and Control (ICPEC), Feb. 2013, pp. 30-34.

[25] K. Ishaque, Z. Salam and H. Taheri, "Accurate MATLAB Simulink PV System Simulator Based on a Two-Diode Model," Journal of Power Electronics, vol. 11, no. 2, pp. 179-187, 2011.

[26] M. Hejri, H. Mokhtari, M. R. Azizian, M. Ghandhari and L. Soder, "On the Parameter Extraction of a Five-Parameter Double-Diode Model of Photovoltaic Cells and Modules," IEEE journal of photovoltaic, vol. 4, no. 3, pp. 915-923, may 2014.

[27] J. Ma, K. L. Man, T. O. Ting, N. Zhang, S. U. Guan and P. W. Wong, "Approximate Single-Diode Photovoltaic Model for Efficient I-V Characteristics Estimation," The Scientific World Journal, vol. 2013, 7 pages, 2013.

[28] A. Necaibia, N. Boutasseta and S. Mouhajer, "A Simple Theoretical Method for the Estimation of Dynamic Resistance in Photovoltaic Panels," International Journal of Computer Applications, vol. 45, no.14, May 2012.

[29] M. Chegaar, Z. Ouennoughi, F. Guechi and H. Langueur, "Determination of Solar Cells Parameters under Illuminated Conditions," Journal of Electron Devices, vol. 2, pp. 17-21, 2003.

[30] N. Enebish, D. Agchbayar, S. Dorjkhand, D. Baatar and I. Ylemj, "Numerical analysis of solar cell current-voltage characteristics," Solar energy materials and solar cells, vol. 29, no. 3, pp. 201-208, 1993.

[31] F. Ghani, M. Duke and J. Carson, "Extraction of solar cell modeling parameters using the Lambert W function," in Australian Solar Energy Society Melbourne, Dec. 2012.
32] J. C. H. Phang, D. S. H. Chan and J. R. Phillips, "Accurate analytical method for the extraction of solar cell," Electronics Letter, vol. 20, no. 10, pp. 406-408, May 1984.

33] M. Zagrouba, A. Sellami, M. Bouaicha and M. Ksouri, "Identification of PV solar cells and modules parameters using the genetic algorithms: application to maximum power extraction," Solar energy, vol. 84, no. 5, pp. 860-866, 2010.

[34] C. Saravanan and M. A. Panneerselvam, "A Comprehensive Analysis for Extracting Single Diode PV Model Parameters by Hybrid GA-PSO Algorithm," International Journal of Computer Applications, vol. 78, no.8, pp. 16-19, September 2013.

[35] H. Qin and J. W. Kimball, "Parameter Determination of Photovoltaic Cells from Field Testing Data using Particle Swarm Optimization," IEEE Power and Energy Conference at Illinois , 2011, pp. 1-4.

[36] M. R. AlRashidi, K. M. El-Naggar and M. F. AlHajri, "Extraction of Photovoltaic Characteristics Using Simulated Annealing," International Conference on Advances in Engineering Sciences and Applied Mathematics, May2014.

[37] M. Ketkar and M. Chopde, "Efficient Parameter Extraction of Solar Cell using Modified ABC," International Journal of Computer Applications, vol. 102, no. 1, Sept. 2014.

[38] D. Oliva, E. Cuevas and G. Pajares, "Parameter identification of solarcells using artificial bee colony optimization," Energy, vol. 72, pp. 93-102, 2014

[39] J. Ma, T. O. Ting, K. L. Man, N. Zhang, S. U. Guan and P. W. Wong, "Parameter estimation of photovoltaic models via cuckoo search," Journal of Applied Mathematics, vol. 2013, 8 pages, 2013.

[40] R. Jovanovic, S. Kais and F. H. Alharbi, "Cuckoo Search Inspired Hybridization of the Nelder-Mead Simplex Algorithm Applied to Optimization of Photovoltaic Cells," arXiv preprint, nov. 2014.

[41] A. Biswas, S. Das, A. Abraham and S. Dasgupta, "Analysis of the reproduction operator in an artificial bacterial foraging system," Applied Mathematics and Computation, vol. 215, no. 9, pp. 33433355, 2010.

[42] J. K. Maherchandani, C. Agarwal and M. Sahi, "Estimation of Solar Cell Model Parameter by Hybrid Genetic Algorithm Using MATLAB," international Journal of Advanced Research in Computer Engineering \& Technology, vol. 1, no. 6, pp. 78-81, August 2012.

[43] Y. del Valle, G. K. Venayagamoorthy, S. Mohagheghi, J. C. Hernandez, and R. G. Harley, "Particle swarm optimization: Basic concepts, variants and applications in power systems," IEEE Transactions on Evolutionary Computation, vol. 12, no. 2, pp. 171195, April 2008.

[44] W. Huang, C. Jiang, L. Xue and D. Song, "Extracting solar cell model parameters based on chaos particle swarm algorithm," International Conference on Electric Information and Control Engineering 2011, IEEE, April 2011, pp. 398-402.

[45] W. Han, H. H. Wang and L. Chen, "Parameters Identification for Photovoltaic Module Based on an Improved Artificial Fish Swarm Algorithm," The Scientific World Journal, 2014.

[46] X. Yuan, Y.He and L. Liu, "Parameter extraction of solar cell models using chaotic asexual reproduction optimization," Neural Computing and Applications, pp. 1-13, 2014.

[47] A. Askarzadeh and A. Rezazadeh, "Parameter identification for solar cell models using harmony search-based algorithms," Solar Energy, vol. 86, no. 11, pp. 3241-3249, 2012.

[48] M. T. Elhagry, A. A. T. Elkousy, M. B. Saleh, T. F. Elshatter and E. M. Abou-Elzahab, "Fuzzy modeling of photovoltaic panel equivalent circuit," in proceedings of the photovoltaic specialists conference, 2000, vol. 15, no. 22, pp.1656-1659.

[49] T. Bendib, F. Djeffal, D. Arar and M. Meguellati, "Fuzzy-logicbased approach for organic solar cell parameters extraction," In Proceedings of world congress on engineering, vol. 2, pp. 11821185,2013

[50] E. Karatepe, M. Boztepe and M. Colak, "Neural network based Solar cell mode," Energy Conversion and Management, vol. 47, no. 9, pp. 1159-1178, 2006

[51] A. Kulaksiz, "ANFIS-based estimation of PV module equivalent parameters: application to a stand-alone PV system with MPPT controller," Turkish Journal of Electrical Engineering \& Computer Sciences, vol. 21, pp. $2127-2140,2013$. 